\title{
A Lost Counterexample and a Problem on Illuminated Polytopes
}

\author{
Ronald F. Wotzlaw* Günter M. Ziegler**
}

August 12, 2009

In a Note added in proof to a 1984 paper, Daniel A. Marcus claimed to have a counterexample to his conjecture that a minimal positively $k$-spanning vector configuration in $\mathbb{R}^{m}$ has size at most $2 \mathrm{~km}$. However, the counterexample was never published, and seems to be lost.

Independently, ten years earlier, Peter Mani in 1974 solved a problem by Hadwiger, disproving that every illuminated $d$-dimensional polytope must have at least $2 d$ vertices.

These two studies are related by Gale duality, an elementary linear algebra technique devised by Micha A. Perles in the sixties. Thus, we note that Mani's study provides a counterexample for Marcus' conjecture with exactly the parameters that Marcus had claimed. In the other direction, with Marcus' tools we provide an answer to a problem left open by Mani: Could illuminated $d$-dimensional polytopes on a minimal number of vertices be nonsimplicial?

\section{Marcus' lost counterexample and Mani's problem}

A positively spanning vector configuration $U$ in $\mathbb{R}^{m}$ is a finite configuration of vectors (multiples are allowed) that positively span $\mathbb{R}^{m}$, that is, if $U=$ $\left\{u_{1}, \ldots, u_{n}\right\}$ and $v \in \mathbb{R}^{m}$, there are real nonnegative numbers $\lambda_{1}, \ldots, \lambda_{n}$ such that

$$
v=\lambda_{1} u_{1}+\lambda_{2} u_{2}+\ldots+\lambda_{n} u_{n} .
$$

A positively $k$-spanning vector configuration is a positive spanning vector configuration that is still positively spanning even if at most $k-1$ vectors are deleted from the configuration.

In two papers [8] 9], dating from 1981 and 1984, Marcus studied properties of positive $k$-spanning vector configurations. In particular, he was interested in upper bounds on the cardinality of minimal positively $k$-spanning vector configurations, that is, of positively $k$-spanning vector configurations that are minimal with respect to inclusion:

Question 1 (Marcus [8] [9]; see also [10]). What is the maximum size of a minimal positively $k$-spanning vector configuration in $\mathbb{R}^{m}$ ?

\footnotetext{
${ }^{*}$ Research supported by the Deutsche Forschungsgemeinschaft (DFG) within the research training group "Methods for Discrete Structures"(GRK 1408)

** Partially supported by DFG
} 
A classical result, known as the Blumenthal-Robinson theorem [1] 3] [13], states that for $k=1$ the exact answer is $2 m$. Marcus conjectured that the answer for the general case is $2 \mathrm{~km}$. This would clearly be best possible: The configuration that consists of $k$ copies of the standard basis vectors and their negatives, that is, $\pm e_{1}, \ldots, \pm e_{m}$, is a minimal positively $k$-spanning vector configuration in $\mathbb{R}^{m}$. Yet for $k \geq 2$ it is not obvious that there is a finite upper bound. However, this was proved by Marcus; it can also be derived from Perles' Skeleton Theorem for (convex) polytopes [6]; see [14.

In the following, we focus on the case $k=2$, which is particularly interesting. It was pointed out and used by Marcus [8] [9] that via Gale diagrams the above question translates into a question about polytopes. Indeed, for $k \geq 2$ every positively $k$-spanning vector configuration on $n$ vectors is a Gale diagram of a $d$-dimensional polytope $P$ on $f_{0}=n$ vertices, where $d=n-m-1$. The main relation between the vector configuration and the polytope that we will use (which have the same size/number of vertices, but live in different dimensions) is that the minimal positively-spanning subconfigurations of vectors correspond exactly to the complements of the vertex sets of facets of the polytope; for details of this construction see, for example, [4, Sect. 5.4] or [15, Lect. 6].

The fact that we look at a minimal positively $k$-spanning vector configuration for $k=2$ translates, via Gale duality, into the following special property of the polytope: For every vertex $u$ of $P$ there is a different vertex $v$ such that $u$ and $v$ are not connected by an edge of $P$, that is, the edge between $u$ and $v$ is missing. In other words, no vertex is connected to all the other vertices. We will call a polytope with this property unneighborly.

With this translation, Question 1 may be rephrased as follows:

Question 2 (Marcus 8, 9]). What is the minimum number of vertices of an unneighborly d-polytope?

Marcus' conjectured bound of $4 m$ for a positively 2-spanning vector configuration in $\mathbb{R}^{m}$ would imply that an unneighborly polytope in dimension $d$ has at least $4(d+1) / 3$ vertices. This is wrong, as we shall see, and it seems that this was noticed by Marcus: In 9], a "Note added in proof" says that he had discovered an unneighborly polytope of dimension $d=36$ on $f_{0}=49$ vertices. (The bound of $4(d+1) / 3$ would imply that such a polytope would need to have at least 50 vertices.) However, there is no mention of a construction for this polytope or any kind of reference so that would help to recover this example. McMullen, who reviewed Marcus' paper for "Mathematical Reviews" and "Zentralblatt" had not seen the counterexample either [11. In the following we will refer to this polytope as Marcus' lost counterexample.

As far as we know, Marcus was not aware of the work Peter Mani had done ten years earlier. He had studied a question by Hugo Hadwiger on illuminated polytopes. These are polytopes in which every vertex lies on an inner diagonal, that is, a segment connecting two vertices that passes through the interior of the polytope. The $d$-dimensional crosspolytope, that is, the polytope generated from the points $\pm e_{1}, \pm e_{2}, \ldots, \pm e_{d}$ is an example for such a polytope on $2 d$ vertices. Clearly, inner diagonals are missing edges, and thus every illuminated 
polytope is also unneighborly. (McMullen [11] proposes to call them strongly unneighborly.)

Question 3 (Hadwiger [5]). What is the minimum number of vertices of an illuminated d-polytope? More specifically, is it $2 d$ ?

In [7, Mani gave a remarkable complete answer to this question. Indeed, the conjectured bound of $2 d$ turned out to be wrong, whereas the correct bound is roughly $d+2 \sqrt{d}$. More precisely, Mani showed that every illuminated $d$ polytope has at least

$$
M(d):=\min \{2 d, d+p(d)+\lceil d / p(d)\rceil+1\}
$$

vertices, where we set $p(d):=\left\lceil\frac{\sqrt{4 d+1}-1}{2}\right\rceil$ for $d \geq 1$. McMullen [11] has noted that one can write the function $M(d)$ in the following simple form:

$$
M(d)=\min \left\{2 d,\left\lceil(\sqrt{d}+1)^{2}\right\rceil\right\}=\min \{2 d, d+1+\lceil 2 \sqrt{d}\rceil\} .
$$

According to Mani, every illuminated polytope has at least $M(d)$ vertices and examples on $M(d)$ vertices exist: They are obtained from a cyclic $d$ polytope with $d+p(d)$ vertices by "stacking" a new vertex on $\lceil d / p(d)\rceil+1$ well-chosen facets. (The operation stacking a facet of a polytope $P$ is performed in the following way: Take a new point that is beyond the facet we want to stack but beneath all other facets. Then take the convex hull of $P$ and the new point. Observe that this creates inner diagonals from the new point to each of the vertices of $P$ that do not lie on the stacked facet.)

In particular, for large enough $d$ there are illuminated - and thus also unneighborly - polytopes on much fewer vertices than $4(d+1) / 3$. Indeed, the smallest $d$ where this occurs is $d=36$, and Mani's illuminated polytope in dimension $d=36$ has $M(36)=49$ vertices, so it has exactly the same parameters as Marcus' lost counterexample. We will probably never know whether this is the same polytope that Marcus had in mind: He never published on the subject again, and when we tried to contact him via the California State Polytechnic University in Pomona, where he had been on the faculty for a number of years, we received the information that he had passed away some years ago.

We will refer to illuminated polytopes on the minimal number of $f_{0}=M(d)$ vertices as Mani polytopes. The Mani polytopes constructed by Mani himself are, by construction, simplicial (that is, all facets are simplices). In relation to Question 3, Mani thus asked:

Question 4 (Mani [7]; see also Bremner \& Klee [2]). Are all illuminated polytopes with the minimum number of vertices simplicial? Or are there nonsimplicial Mani polytopes?

Below we give a complete answer to this question: Up to dimension 5, all Mani polytopes are simplicial, and there is only one combinatorial type, given by the crosspolytope. For every $d \geq 6$ though, we construct a nonsimplicial Mani polytope on the minimum number of vertices. This corrects a statement by Bremner \& Klee [2, who had claimed that up to dimension 7 all extremal illuminated polytopes were crosspolytopes. 
Thus our construction that solves Mani's question is based on a Gale diagram construction, thus on a construction that uses Marcus' positively $k$ spanning vector configurations. In turn, Marcus' conjecture on the size of minimal positively $k$-spanning vector configurations is refuted by taking Mani's viewpoint of illuminated polytopes.

To summarize, the precise answers for Questions 11 and 2 remain open, although Marcus' conjectured answers are refuted by Mani's construction as well as by our construction in this paper: They yield illuminated $d$-polytopes on roughly $d+2 \sqrt{d}$ vertices, while Marcus' work implies that an unneighborly $d$-polytope has at least roughly $d+\sqrt{2 d}$ vertices. Question 3 was solved by Mani [7, and Question 4 is solved by our construction.

Marcus' conjecture for Question 1 is proven wrong for all $k \geq 2$ in the first author's PhD thesis [14] based on Mani's construction for the case $k=2$.

We briefly introduce some notation. If $P$ is a $d$-polytope (which in the following will always be assumed to be full-dimensional, that is, embedded in $\mathbb{R}^{d}$ ), we denote by $\operatorname{vert}(P)$ the set of vertices and by $f_{0}=f_{0}(P)=|\operatorname{vert}(P)|$ the number of vertices. For polytope terminology we refer to [4] and [15].

\section{Unique Mani polytopes}

We show that in dimensions $1 \leq d \leq 5$ Mani polytopes are combinatorially unique. Thus the only combinatorial type that appears is the $d$-crosspolytope. Our argument is mainly based on the original results by Mani [7] and the subsequent simplification by Rosenfeld [12].

Definition (Self illuminated, opposite sets). Let $P$ be an illuminated $d$-polytope. A set of vertices $U \subseteq \operatorname{vert}(P)$ is said to illuminate itself if for every vertex $v \in U$ there is a vertex $u \in U$ such that

$$
[u, v]:=\left\{x \in \mathbb{R}^{d}: x=\lambda u+(1-\lambda) v, \lambda \in[0,1]\right\}
$$

is an inner diagonal.

A set $W \subseteq \operatorname{vert}(P)$ is said to lie opposite the vertex $v \in \operatorname{vert}(P)$ if for every $w \in W$ the segment $[v, w]$ is an inner diagonal and $\operatorname{vert}(P) \backslash(W \cup\{v\})$ illuminates itself.

Let $\Gamma(P):=\max \{|W|: W$ lies opposite some $v \in \operatorname{vert}(P)\}$.

The following is a slightly stronger statement than the main result from [12] that is easily extracted from Rosenfeld's proof.

Lemma 1 (Rosenfeld [12]). Let $P$ be an illuminated d-polytope. If $\Gamma(P)=1$, then $f_{0} \geq 2 d$ and there is a perfect matching on the inner diagonals, that is, there are pairwise vertex-disjoint inner diagonals that cover all vertices of $P$.

The next lemma is easily derived from results by Mani; just combine the statements in [7, Lemma 1, Proposition 2, and Proposition 3].

Lemma 2 (Mani [7]). Let $d \geq 3$, let $P$ be a Mani d-polytope, and assume that $\Gamma(P) \geq 2$. Then $f_{0}(P) \geq d+p(d)+\lceil d / p(d)\rceil+1$. 
Corollary 3. Let $d \geq 3$, let $P$ be a Mani d-polytope with $\Gamma(P) \geq 2$. Then $d \geq 6$.

Proof. By Lemma 2, the number of vertices is at least $d+p(d)+\lceil d / p(d)\rceil+1$. But for $3 \leq d \leq 5$ we have that $d+p(d)+\lceil d / p(d)\rceil+1>2 d$. Since $P$ is a Mani polytope we must have $d \geq 6$.

Theorem 4. For $1 \leq d \leq 5$ there is exactly one combinatorial type of Mani d-polytope, namely the d-dimensional crosspolytope.

Proof. The cases $d=1,2$ are trivial, so assume $d \geq 3$. Let $P$ be a Mani $d$ polytope with $3 \leq d \leq 5$. By Corollary 3 , we have $\Gamma(P)=1$. Lemma 1 and the existence of the crosspolytopes then imply that $f_{0}(P)=2 d$ and that there is a perfect matching on the inner diagonals. Since any facet of $P$ can contain only one vertex of any inner diagonal, the set of facets is a subset of the facets of the $d$-crosspolytope. This implies that $P$ is the $d$-crosspolytope.

\section{$3 \quad$ Nonsimplicial Mani polytopes}

Theorem 5. There exists a nonsimplicial Mani d-polytope for every $d \geq 6$.

Proof. For every $d \geq 6$ we construct a nonsimplicial Mani $d$-polytope. (Observe that for $d=6,7$ we have $d+p(d)+\lceil d / p(d)\rceil+1=2 d$.) Let $p \geq 1, q:=\lceil d / p\rceil$, and choose an $\ell$ with $1 \leq \ell \leq q-1$.

We construct a nonsimplicial polytope $Q$ on $d+p$ vertices that has $q+1$ simplex facets, such that stacking onto these facets produces a nonsimplicial illuminated $d$-polytope. (What we describe here is in fact a whole family of such polytopes, indexed by the parameter $\ell$.)

We describe $Q$ in terms of a Gale diagram $A$. Let

$$
B=\left\{-\mathbf{1}, e_{1}, \ldots, e_{p-1}\right\},
$$

where 1 denotes the vector in which all entries are 1 . This is a positive basis of $\mathbb{R}^{p-1}$ of cardinality $p$. The vectors in $A$ are the following:

(1) Take $\ell$ copies of $B$, and denote them by $B_{1}, \ldots, B_{\ell}$.

(2) Take $q-\ell$ copies of $-B$, and denote them by $\tilde{B}_{1}, \ldots, \tilde{B}_{q-\ell}$.

(3) Furthermore, take the vectors $\mathbf{1},-e_{1}, \ldots,-e_{d+p-p q-1}$.

Then the number of vectors in $A$ is $d+p$.

By the translation between Gale diagram and polytope combinatorics, every $B_{i}, i=1, \ldots \ell$, and every $\tilde{B}_{j}, j=1, \ldots, q-\ell$ corresponds to a facet complement of size $p$ in $Q$, that is, to the complement of a simplex facet. If we augment the set $\left\{\mathbf{1},-e_{1}, \ldots,-e_{d+p-p q-1}\right\}$ to a positive basis $B^{\prime}$ by taking the last $p q-d$ vectors of $\tilde{B}_{1}$, we get that

$$
\left\{B_{i}: i=1, \ldots, \ell\right\} \cup\left\{\tilde{B}_{j}: j=1, \ldots, q-\ell\right\} \cup\left\{B^{\prime}\right\}
$$

is a set of subconfigurations of $A$ that correspond to complements of simplex facets of $Q$. These complements cover all vertices of $Q$. Thus, stacking onto the corresponding facets we obtain an illuminated polytope $P$ on $d+p+q+1$ 

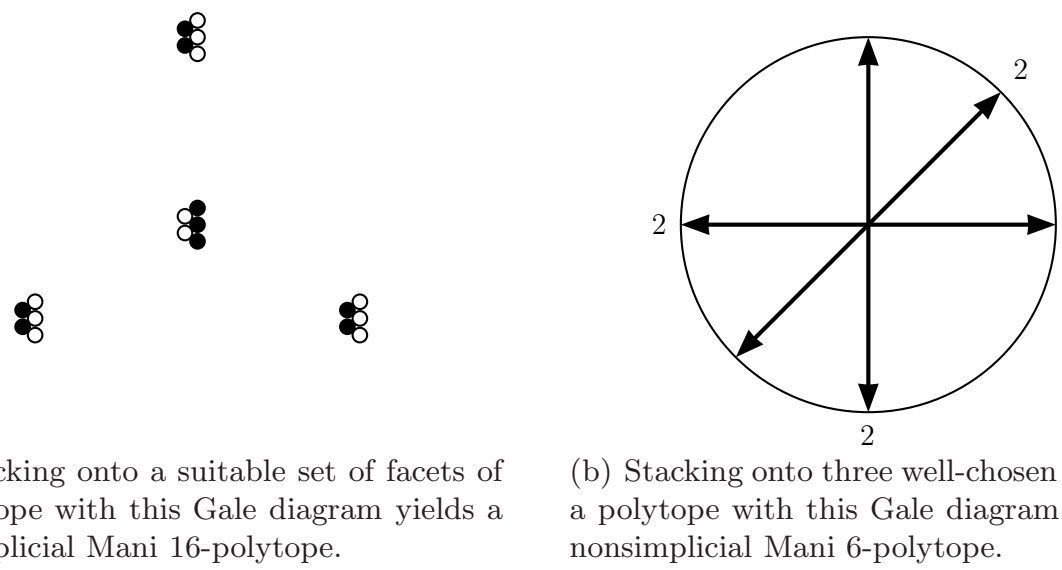

(a) Stacking onto a suitable set of facets of a polytope with this Gale diagram yields a nonsimplicial Mani 16-polytope.

(b) Stacking onto three well-chosen facets of a polytope with this Gale diagram yields a nonsimplicial Mani 6-polytope.

Figure 1: Gale diagrams of building blocks for nonsimplicial Mani polytopes.

vertices, since every vertex will be on an inner diagonal to one of the stacking vertices. For $p:=p(d)$ we get that $P$ is a Mani polytope.

If $1 \leq \ell \leq q-1$, then there is a set of two opposite vectors in $A$, which corresponds to a facet complement, so $Q$ is nonsimplicial unless $p=2$. For $d \geq 7$, we have $p(d) \geq 3$ and we indeed get a nonsimplicial polytope. However, for $d=6$ we get $p=p(d)=2$ and $q=3$. In this case, we choose $p:=3$ instead of $p:=p(d)$. Then $q=\lceil d / p\rceil=2$ and we have $f_{0}(P)=12=M(6)$, that is, $P$ is a Mani polytope.

In both cases, the polytope $P$ is nonsimplicial, because $Q$ is nonsimplicial and we only stack onto simplex facets.

We look at two examples that arise from the above description.

Example 6. For $d=16, p=p(d)=4, q=4$, and $\ell=3$, we display the result of the construction as an "affine Gale diagram" in Figure 1(a), where all points in a cluster represent distinct copies of a single point. (See [15, Chapter 6] for affine Gale diagrams and how they are related to ordinary Gale diagrams.)

In this case, the polytope $Q$ has $f_{0}=20$ and five disjoint simplex facet complements of size four that cover all vertices. This yields a nonsimplicial illuminated 16-polytope on $M(16)=25$ vertices.

Example 7. For the special case $d=6$, we get a polytope $Q$ as in the proof of Theorem 5 by constructing the Gale diagram in Figure 1(b) with $p=3, q=2$, and $\ell=1$.

The Gale diagram has three disjoint positive bases that cover all vectors: the basis $B_{1}=\left\{-\mathbf{1}, e_{1}, e_{2}\right\}$, and the bases $\tilde{B}_{1}=B^{\prime}=\left\{\mathbf{1},-e_{1},-e_{2}\right\}$. These bases correspond to complements of simplex facets of $Q$. Stacking onto these three facets produces a nonsimplicial illuminated 6-polytope on $M(6)=12$ vertices.

Acknowledgements. The authors thank Raman Sanyal for bringing Mani's paper to their attention. Peter McMullen provided valuable comments during a number of email exchanges in 2008 and 2009. 


\section{References}

[1] L. M. Blumenthal, Theory and Applications of Distance Geometry, Oxford, 1953.

[2] D. Bremner and V. Klee, Inner diagonals of convex polytopes, J. Combin. Theory Ser. A, 87 (1999), pp. 175-197.

[3] C. Davis, Theory of positive linear dependence, Amer. J. Math. 76 (1954), pp. $733-746$.

[4] B. Grünbaum, Convex Polytopes, vol. 221 of Graduate Texts in Mathematics, Springer-Verlag, New York, 2003. Second edition by V. Kaibel, V. Klee and G. M. Ziegler (original edition: Interscience, London 1967).

[5] H. Hadwiger, Ungelöste Probleme, Nr. 55, Elemente Math., 27 (1972), p. 57.

[6] G. Kalai, Some aspects of the combinatorial theory of convex polytopes, in Polytopes: Abstract, Convex and Computational (Scarborough, ON, 1993), vol. 440 of NATO Adv. Sci. Inst. Ser. C Math. Phys. Sci., Kluwer Acad. Publ., Dordrecht, 1994, pp. 205-229.

[7] P. Mani, Inner illumination of convex polytopes, Comm. Math. Helv., 49 (1974), pp. 65-73.

[8] D. A. Marcus, Minimal positive 2-spanning sets of vectors, Proc. Amer. Math. Soc. 82 (1981), pp. 165-172.

[9] - Gale diagrams of convex polytopes and positive spanning sets of vectors, Discrete Appl. Math. 9 (1984), pp. 47-67.

[10] P. McMullen, Transforms, diagrams and representations, in Contributions to Geometry: Proc. Geometry Symposium in Siegen 1978, J. Tölke and J. Wills, eds., Birkhäuser Verlag, Basel, 1979, pp. 92-130.

[11] — 2008, 2009. Personal communications.

[12] M. Rosenfeld, Inner illumination of convex polytopes, Elem. Math., 30 (1974), pp. 27-28.

[13] G. C. Shephard, Diagrams for positive bases, J. London Math. Soc., 4 (1971), pp. 165-175.

[14] R. F. WotzLaw, Incidence Graphs and Unneighborly Polytopes, PhD thesis, Technische Universität Berlin, 2009. Published online at http://opus.kobv.de/tuberlin/volltexte/2009/2221/.

[15] G. M. Ziegler, Lectures on Polytopes, vol. 152 of Graduate Texts in Mathematics, Springer-Verlag, 1995.

Ronald F. Wotzlaw, MA 6-2, Inst. Mathematics, TU Berlin, 10623 Berlin, GERMANY

E-mail address: wotzlaw@math.tu-berlin.de

Günter M. Ziegler, MA 6-2, Inst. Mathematics, TU Berlin, 10623 Berlin, GERMANY

E-mail address: ziegler@math.tu-berlin.de 